\title{
Reduction and regularization of the Kepler problem
}

\section{J. C. van der Meer $^{1}$ iD}

Received: 9 July 2020 / Revised: 5 June 2021 / Accepted: 7 June 2021 /

Published online: 8 July 2021

(c) The Author(s) 2021

\begin{abstract}
The KS regularization connects the dynamics of the harmonic oscillator to the dynamics of bounded Kepler orbits. Using orbit space reduction, it can be shown that reduced harmonic oscillator orbits can be identified with re-parametrized Kepler orbits by factorizing the KS map as reduction mapping followed by a chart on the reduced phase space. In this note, we will show that also other regularization maps can be obtained this way. In particular, we will show how Moser's regularization and Ligon-Schaaf regularization are related to KSregularization. All regularizations are a result of choosing the right invariants to represent the reduced phase space, which is isomorphic to $T^{+} S^{3}$, and a chart on this reduced phase space. We show how this opens the way to directly reduce the KS transformed Kepler system and find other regularization maps that are valid for all values of the Keplerian energy similar to Ligon-Schaaf regularization.
\end{abstract}

Keywords Geometric reduction · Harmonic oscillator · Kepler problem · Regularization

Mathematics Subject Classification 53D20 $\cdot 37 \mathrm{~J} 15 \cdot 70 \mathrm{H} 05 \cdot 70 \mathrm{H} 33$

\section{Introduction}

The Kustaanheimo-Stiefel regularization is a well-known regularizing transformation for the equations of Kepler motion in three-dimensional space. The purpose of this regularization is to remove the existing singularity at the origin of the coordinate system which corresponds to collision orbits. Ideas about regularizing this problem go back to Euler who considered the one-dimensional problem of the collision of two bodies and to Levi-Civita (1906) who considered the two-dimensional variant. In Kustaanheimo (1964) proposed an extension to the Levi-Civita regularization in four dimensions based on spinors and this idea was expanded upon in Kustaanheimo and Stiefel (1965) by both Kustaanheimo and Stiefel. Later Stiefel and Scheifele would give a more complete and formal treatment in Stiefel and Scheifele (1971).

\footnotetext{
$凶$ J. C. van der Meer j.c.v.d.meer@tue.nl

1 Faculteit Wiskunde en Informatica, Technische Universiteit Eindhoven, Eindhoven, The Netherlands
} 
The full KS-regularization procedure usually consists of three steps. Starting with the Kepler problem in $\mathbb{R}^{6}$ one considers a fixed energy surface, applies a time re-scaling, and applies the KS-map to finally obtain the harmonic oscillator on $\mathbb{R}^{8}$. The KS-map increases the number of degrees of freedom by one which is caused by the introduction of a free angle. In this paper, we will also focus on other regularizations. Often fixing the energy and re-scaling time is a necessary step.

Although there are many ways to describe the KS transformation using angles (Stiefel and Scheifele 1971; Ferrer and Crespo 2018), or quaternions (Vivarelli 1983; Waldvogel 2006), we will choose to use reduction of the harmonic oscillator on $\mathbb{R}^{8}$. Identifying $\mathbb{R}^{8}$ with $\mathbb{H} \times \mathbb{H}$, one can easily make the step to quaternions. The classical KS transformation connects the Kepler flow on $\mathbb{R}^{6}$ to the harmonic oscillator flow on $\mathbb{R}^{8}$ and raises the dimension by introducing a free angle (Kustaanheimo and Stiefel 1965). Consequently a whole torus of periodic solutions of the harmonic oscillator corresponds to just one bounded Kepler orbit under the KS transformation. Orbit space reduction with respect to the $S^{1}$-action corresponding to this free angle, i.e., the action corresponding to the bilinear relation, will map $\mathbb{R}^{8}$ to a six-dimensional manifold embedded in $\mathbb{R}^{16}$. Under this reduction, the harmonic oscillator flow on the torus reduces to just one circular orbit that can be identified with a bounded Kepler orbit. We will show that the six-dimensional reduced phase space is a manifold diffeomorphic to $T^{+} S^{3}=\left\{(u, v) \in \mathbb{R}^{8}:|u|=1,\langle u, v\rangle=0, v \neq 0\right\}$ in Sect. 3. To obtain a map from $\mathbb{R}^{8}$ to $\mathbb{R}^{6}$, i.e., a KS-transformation, we will have to choose an appropriate chart for this manifold. How to obtain the classical KS-transformation was shown in van der Meer (2015). It will be introduced in Sect. 2. Note that the role of $T^{+} S^{3}$ in the regularization of the Kepler problem became clear through Moser's regularization (Moser 1958). Kummer (1982) establishes a relation between Moser's and KS regularization showing that the "completed" phase space of the Kepler problem is diffeomorphic to $T^{+} S^{3}$. Kummer constructs his maps in terms of "generators" of the group actions involved in the same way as we use the invariants to define the orbit map.

The reduction will be performed using constructive geometric reduction, or orbit space reduction, by explicitly constructing an orbit map using a Hilbert basis of invariants for the $S^{1}$-action, a method of reduction explained in van der Meer (1985). In this construction, there is a lot of freedom, many choices will lead to the same result. The possibilities leading to a chart in which one obtains the Kepler system will be described for the main part in Sect. 2. Interesting is that Stiefel and Scheifele precede their choice of the map, that was later called the KS-map, by "for example." Also in many other papers the non-uniqueness is considered. For instance, in Breiter and Langner (2017), it is shown that one can define a KS-map using any unit quaternion. We will show that this is related to the $S O(3)$ symmetry of the Kepler problem which is the reduced action of an $S O$ (3) symmetry of the harmonic oscillator leaving the harmonic oscillator Hamiltonian and the bilinear relation invariant. Furthermore, in Ferrer and Crespo (2018), it is mentioned that there are more possibilities for the bilinear relation.

KS-regularization is just one of the possibilities to regularize the Kepler problem. There is also Moser's regularization (Moser 1958), based on the stereographic projection of the threesphere, and relating the Kepler flow to the geodesic flow, and Ligon-Schaaf regularization (Ligon and Schaaf 1976) relating the Kepler flow to the flow of the Delaunay Hamiltonian (see also Cushman and Bates 2015), which is also a re-parametrization of the geodesic flow. Note that Moser's map, as well as the Ligon-Schaaf map, are defined on $T^{+} S^{3} \subset \mathbb{R}^{8}$. Thus, the dynamics of the obtained systems on $\mathbb{R}^{8}$ has to be constrained to $T^{+} S^{3}$ to obtain the regularized system. In both cases, this constrained flow is not only a re-parametrization of the geodesic flow but also a re-parametrization of the harmonic oscillator flow. Therefore, 
these regularizations are only different with respect to choosing the invariants for the orbit map.

In Sect. 4, we will consider Moser's regularization in the context of orbit space reduction. In this section, it is shown that Moser's regularization can be connected to KS-regularization by a map on the codomain of the orbit map, that is by changing the invariants defining the orbit map. The obtained relation is similar to the one obtained by Kummer (1982). In both KS and Moser's regularization, restriction to an energy level and time re-scaling is part of the process.

In Sect. 5, we will review the Ligon-Schaaf map (Ligon and Schaaf 1976; Ligon 1973, 2018; Cushman and Duistermaat 1997; Heckman and De Laat 2012; Cushman and Bates 2015). The LS-map uses an energy-dependent version of Moser's map and maps the Kepler system to the Delaunay Hamiltonian on $T^{+} S^{3}$. This regularization has the advantage that it avoids restriction to an energy level and time re-scaling. However, it has the drawback that the inverse LS-map contains an angle that is only implicitly defined. The LS-map can also be put in the framework of reduction and connected to the KS map.

In Sect. 6, we will introduce a scaled version, i.e., energy-dependent version, of the reduction valid for all negative Keplerian energies. Formulating the Kepler Hamiltonian on $\mathbb{R}^{8}$ using the KS-map and using the reduction associated with the LS-map, one can obtain the constrained Delaunay system as a reduction of the Kepler system. Some alternative reductions and corresponding regularizations are suggested in Sect. 7, where the positive energy case is considered as well.

Due to the vast amount of literature about the Kepler problem, we do not pretend to be complete in our references. For a discussion of references relating reduction and KS regularization, we refer to van der Meer (2015). In addition to this also Cordani (2003) has to be mentioned as here also the relation between reduction and KS regularization, and the role of $T^{+} S^{3}$ is studied. Nice bibliographies can be found in Cordani (2003), Efstathou and Sadovskii (2010).

\section{The KS transformation and reduction}

Let us start this section with the first step in the regularization process, the time re-scaling. Consider the Kepler Hamiltonian for negative energy

$$
K(x, y)=\frac{1}{2}|y|^{2}-\frac{1}{|x|}=-\frac{1}{2} k^{2}, k>0 .
$$

We construct the pre-regularized Hamiltonian

$$
\hat{K}(x, y)=\frac{|x|}{k}\left(K(x, y)+\frac{1}{2} k^{2}\right)+\frac{1}{k}=\frac{1}{2 k}|x|\left(|y|^{2}+k^{2}\right) .
$$

The energy level $K(x, y)=-\frac{1}{2} k^{2}$ corresponds to the energy level $\hat{K}(x, y)=\frac{1}{k}$. The Hamiltonian vector field corresponding to $\hat{K}$ is

$$
\begin{aligned}
& \frac{\mathrm{d} x}{\mathrm{~d} s}=\frac{1}{k}|x| \frac{\partial K}{\partial y}+\left(K(x, y)+\frac{1}{2} k^{2}\right) \frac{\partial}{\partial y} \frac{|x|}{k}, \\
& \frac{\mathrm{d} y}{\mathrm{~d} s}=-\frac{1}{k}|x| \frac{\partial K}{\partial x}-\left(K(x, y)+\frac{1}{2} k^{2}\right) \frac{\partial}{\partial x} \frac{|x|}{k} .
\end{aligned}
$$


On $K(x, y)=-\frac{1}{2} k^{2}$, this corresponds to

$$
\frac{\mathrm{d} x}{\mathrm{~d} s}=\frac{1}{k}|x| \frac{\partial K}{\partial y}, \frac{\mathrm{d} y}{\mathrm{~d} s}=-\frac{1}{k}|x| \frac{\partial K}{\partial x} .
$$

With $\frac{\mathrm{d} s}{\mathrm{~d} t}=\frac{k}{|x|}$, this is a time re-scaled version, or re-parametrization, of the Kepler vector field. It is this time re-scaling which actually makes it possible to map to a regular system when using classical regularization. The integral curves of the pre-regularized Hamiltonian vector field corresponding to $\hat{K}$ with energy $\frac{1}{k}$ agree with the integral curves of the Kepler vector field with energy $-\frac{1}{2} k^{2}$. The vector fields are equivalent, but not symplectically conjugate, as one is a re-parametrization of the other, where the re-parametrization depends on the variables.

In van der Meer (2015), the KS map was described as a chart for the reduced phase space obtained by reducing $\mathbb{R}^{8}$ with respect to an $S^{1}$ action that was related to the bilinear relation. When one considers the harmonic oscillator on $\mathbb{R}^{8}$, then the harmonic oscillator reduces to the pre-regularized Kepler problem (i.e., the Kepler problem after time re-scaling and on a fixed energy-level) within this chart on the reduced phase space. For sake of completeness, we will repeat the procedure below and in addition show that the procedure is independent of the choice of the orbit map.

Consider $\mathbb{R}^{8}$ with coordinates $(q, Q)$. The Hamiltonian for the harmonic oscillator is $H_{2}(q, Q)=\frac{1}{2}|q|^{2}+\frac{1}{2}|Q|^{2},|$.$| denoting the Euclidean norm on \mathbb{R}^{4}$. Furthermore, let $\Xi(q, Q)=\left(q_{1} Q_{2}-q_{2} Q_{1}\right)+\left(q_{3} Q_{4}-q_{4} Q_{3}\right)$. Note that we use the notation of van der Meer (2015), where for the formulation of the KS-map we followed Cushman and Bates (2015).

The invariants for the flow of $\Xi$ are (see Egea 2007)

$$
\begin{aligned}
s_{1} & =q_{1}^{2}+q_{2}^{2} \quad s_{2}=q_{3}^{2}+q_{4}^{2} \\
s_{3} & =Q_{1}^{2}+Q_{2}^{2} \quad s_{4}=Q_{3}^{2}+Q_{4}^{2} \\
s_{5} & =q_{1} Q_{1}+q_{2} Q_{2} \quad s_{6}=q_{3} Q_{3}+q_{4} Q_{4} \\
s_{7} & =q_{1} Q_{2}-q_{2} Q_{1} \quad s_{8}=q_{3} Q_{4}-q_{4} Q_{3} \\
s_{9} & =q_{1} q_{4}-q_{2} q_{3} \quad s_{10}=q_{1} q_{3}+q_{2} q_{4} \\
s_{11} & =Q_{1} Q_{4}-Q_{2} Q_{3} \quad s_{12}=Q_{1} Q_{3}+Q_{2} Q_{4} \\
s_{13} & =q_{1} Q_{4}-q_{2} Q_{3} \quad s_{14}=q_{1} Q_{3}+q_{2} Q_{4} \\
s_{15} & =Q_{1} q_{4}-Q_{2} q_{3} \quad s_{16}=Q_{1} q_{3}+Q_{2} q_{4} .
\end{aligned}
$$

The orbit map for the $\Xi$-action is therefore

$$
\tau_{\Xi}: \mathbb{R}^{8} \rightarrow \mathbb{R}^{16} ;(q, Q) \rightarrow\left(s_{1}, \ldots, s_{16}\right) .
$$

The image of $\tau_{\Xi}$ is the $\Xi$-orbit space, which is obtained by dividing out the $S^{1}$-action generated by $\Xi$, and thus of dimension seven. Restricting to the energy level $\Xi(q, Q)=c$ then gives the reduced phase space and reduces the dimension to six. Thus, $V_{c}=$ $\tau_{\Xi}\left(\Xi^{-1}(c)\right), c \in \mathbb{R}$ is a $\Xi$-reduced phase space. Reduced phase spaces are, in general, semi-algebraic sets. On the six-dimensional reduced phase space $\tau_{\Xi}\left(\Xi^{-1}(0)\right)$, we may choose the chart

$$
\begin{aligned}
& x_{1}=2 s_{10}=2\left(q_{1} q_{3}+q_{2} q_{4}\right) \\
& x_{2}=2 s_{9}=2\left(q_{1} q_{4}-q_{2} q_{3}\right) \\
& x_{3}=s_{1}-s_{2}=\left(q_{1}^{2}+q_{2}^{2}\right)-\left(q_{3}^{2}+q_{4}^{2}\right),
\end{aligned}
$$


Fig. 1 Diagram relating $\Xi$-reduction and the KS-map

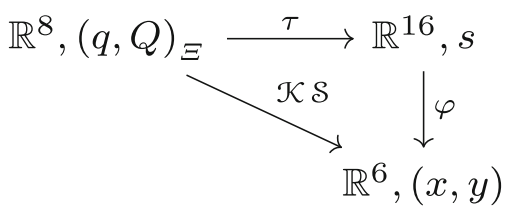

$$
\begin{aligned}
& y_{1}=\frac{1}{s_{1}+s_{2}}\left(s_{14}+s_{16}\right)=\frac{q_{1} Q_{3}+q_{2} Q_{4}+Q_{1} q_{3}+Q_{2} q_{4}}{q_{1}^{2}+q_{2}^{2}+q_{3}^{2}+q_{4}^{2}}, \\
& y_{2}=\frac{1}{s_{1}+s_{2}}\left(s_{13}+s_{15}\right)=\frac{q_{1} Q_{4}-q_{2} Q_{3}+Q_{1} q_{4}-Q_{2} q_{3}}{q_{1}^{2}+q_{2}^{2}+q_{3}^{2}+q_{4}^{2}}, \\
& y_{3}=\frac{1}{s_{1}+s_{2}}\left(s_{5}-s_{6}\right)=\frac{q_{1} Q_{1}+q_{2} Q_{2}-q_{3} Q_{3}-q_{4} Q_{4}}{q_{1}^{2}+q_{2}^{2}+q_{3}^{2}+q_{4}^{2}} .
\end{aligned}
$$

The expressions in (3) define exactly one of the representations of the KS-transformation. More precisely, if we define $\varphi: \mathbb{R}^{16} \rightarrow \mathbb{R}^{6}$ to be the map such that $\varphi\left(s_{1}, \ldots, s_{16}\right)=$ $(x(s), y(s))$, then the KS map $\mathcal{K S}:(q, Q) \rightarrow(x, y)$ is $\mathcal{K S}=\varphi \circ \tau_{\Xi}$ (see Fig. 1).

Note that this chart is not a global chart. It is not defined when $s_{1}=s_{2}=0$, or $|q|=0$, which implies that all $s_{i}$ are zero except $s_{3}, s_{4}, s_{11}, s_{12}$, amongst which we have the relation $s_{3} s_{4}=s_{11}^{2}+s_{12}^{2}$.

As we reduced with respect to $\Xi$ we still have a non-trivial $H_{2}$-action on the reduced phase space. In order to compute the $\mathrm{H}_{2}$ vector field in the chart, we have to consider the induced Poisson bracket.

Consider $C^{\infty}\left(\mathbb{R}^{8}, \mathbb{R}\right)$ with standard Poisson bracket $\{$,$\} . When we consider an orbit$ map like $\tau_{\Xi}$ then such a map induces a bracket on $C^{\infty}\left(\mathbb{R}^{16}, \mathbb{R}\right)$ given by $\{f(s), g(s)\}_{\mathbb{R}^{16}}=$ $\{f(s(q, Q)), g(s(q, Q))\}$. This makes the orbit mapping into a Poisson map, that is,

$$
\left\{f \circ \tau_{\Xi}, g \circ \tau_{\Xi}\right\}=\{f, g\}_{\mathbb{R}^{16}} \circ \tau_{\Xi},
$$

for $f, g$ smooth functions on $\mathbb{R}^{16}$. Note that functions $f \circ \tau_{\Xi}$ are the functions invariant under the Hamiltonian flow of the function $\Xi$.

In the same way, the coordinate chart $\varphi$ is a Poisson map,

$$
\{f, g\}_{\mathbb{R}^{6}} \circ \varphi=\{f \circ \varphi, g \circ \varphi\}_{\mathbb{R}^{16}} .
$$

Consequently $\varphi \circ \tau_{\Xi}$ is a Poisson map. Because the standard Poisson structure is nondegenerate, in this case Poisson is equivalent to symplectic.

The reduced vector field for the harmonic oscillator given by $\mathrm{H}_{2}$ can now be given in terms of the induced Poisson bracket

$$
\begin{aligned}
& \left\{x_{1}, H_{2}\right\}=2|x| y_{1}, \\
& \left\{x_{2}, H_{2}\right\}=2|x| y_{2}, \\
& \left\{x_{3}, H_{2}\right\}=2|x| y_{3}, \\
& \left\{y_{1}, H_{2}\right\}=-2 \frac{H_{2}}{|x|^{2}} x_{1}-2 \frac{\Xi}{|x|^{2}}\left(s_{15}-s_{13}\right), \\
& \left\{y_{2}, H_{2}\right\}=-2 \frac{H_{2}}{|x|^{2}} x_{2}-2 \frac{\Xi}{|x|^{2}}\left(s_{14}-s_{16}\right), \\
& \left\{y_{3}, H_{2}\right\}=-2 \frac{H_{2}}{|x|^{2}} x_{3}-2 \frac{\Xi}{|x|^{2}}\left(s_{8}-s_{7}\right),
\end{aligned}
$$


which on $\Xi=0$ and $H_{2}=1$ is precisely two times the pre-regularized (time-re-scaled) Kepler vector field corresponding to $\hat{K}$ as given by (2), with $k=1$. Because of the factor two, the coordinates $x, y$ are the physical coordinates of the Kepler problem up to a re-scaling. Its orbits are re-parametrizations of the Kepler orbits on the level set $K(x, y)=-\frac{1}{2}$ and are the image of harmonic oscillator orbits on the reduce phase space $\tau_{\Xi}\left(\Xi^{-1}(0)\right)$.

The factorization of the KS-map defined in (3) can be obtained for any choice of a basis of invariants defining the orbit map $\tau_{\Xi}$ for the $\Xi$-action.

Consider a diffeomorphism $\delta: \mathbb{R}^{16} \rightarrow \mathbb{R}^{16} ;\left(s_{1}, \ldots, s_{16}\right) \rightarrow\left(\delta_{1}(s), \ldots \delta_{16}(s)\right)$. Then the coordinate chart on the image of $\delta$ becomes $\varphi_{\delta}$ such that $\varphi_{\delta} \circ \delta=\varphi$. Consequently $\varphi_{\delta} \circ \delta \circ \tau_{\Xi}(q, Q)=\varphi \circ \tau_{\Xi}(q, Q)=(x(q, Q), y(q, Q))$ on $\Xi^{-1}(0)$.

Note that any diffeomorphism on the target space of the orbit map provides a new orbit map and that all possible orbit maps are obtained this way. In general, one will reduce with respect to a compact group action, in which case one may choose a Hilbert basis of homogeneous polynomials.

Remark 1 From the above, it is clear that KS maps are not unique. On can apply an arbitrary symplectic diffeomorphism to $\mathbb{R}^{8}$ such that the composition of this map with the KS map gives another representation of the KS map, which can be factorized through the reduction map and chart as before.

The group of symplectic diffeomorphisms that are linear and leave $\Xi$ and $H_{2}$ invariant is $S O$ (4). When we consider $S O(3) \subset S O(4)$, we obtain KS-maps with an arbitrary defining vector as in Breiter and Langner (2017).

If we choose linear symplectic diffeomorphisms that leave $H_{2}$ invariant, we obtain a KS map with a changed bilinear relation, which explains the remark in Ferrer and Crespo (2018) concerning the possibility of multiple choices for the bilinear relation.

\section{The $\Xi-$ reduced phase space}

In this section, we will determine the nature of the reduced phase space for the $\Xi$-action which is given by $\tau_{\Xi}\left(\Xi^{-1}(0)\right)$. Note that the representation of the image of the orbit map $\tau_{\Xi}$ depends on the choice of invariants one needs to construct the orbit map. However, all representations are diffeomorphic.

Introduce the following set of $\Xi$ invariants as in van der Meer (2015).

$$
\begin{aligned}
K_{1}=\frac{1}{2}\left(-s_{1}-s_{3}+s_{2}+s_{4}\right), & L_{1}=-s_{7}+s_{8}, \\
K_{2}=-s_{9}-s_{11}, & L_{2}=s_{14}-s_{16}, \\
K_{3}=-s_{10}-s_{12}, & L_{3}=s_{15}-s_{13}, \\
\Xi=s_{7}+s_{8}, & H_{2}=\frac{1}{2}\left(s_{1}+s_{2}+s_{3}+s_{4}\right),
\end{aligned}
$$

and

$$
\begin{aligned}
2 U_{1}=-\left(s_{5}+s_{6}\right), & V_{1} & =\frac{1}{2}\left(s_{1}+s_{2}-s_{3}-s_{4}\right), \\
U_{2}=s_{10}-s_{12}, & V_{2} & =s_{14}+s_{16}, \\
U_{3}=s_{9}-s_{11}, & V_{3} & =s_{13}+s_{15}, \\
U_{4}=\frac{1}{2}\left(s_{1}-s_{2}-s_{3}+s_{4}\right), & V_{4} & =s_{5}-s_{6} .
\end{aligned}
$$


We have the following relations

$$
\begin{aligned}
U_{1}^{2}+U_{2}^{2}+U_{3}^{2}+U_{4}^{2} & =H_{2}^{2}-\Xi^{2}, \\
V_{1}^{2}+V_{2}^{2}+V_{3}^{2}+V_{4}^{2} & =H_{2}^{2}-\Xi^{2}, \\
U_{1} V_{1}+U_{2} V_{2}+U_{3} V_{3}+U_{4} V_{4} & =0,
\end{aligned}
$$

and

$$
\begin{array}{ll}
V_{1} U_{2}-U_{1} V_{2}+H_{2} K_{3}-L_{3} \Xi=0, & V_{2} U_{3}-U_{2} V_{3}+H_{2} L_{1}-K_{1} \Xi=0, \\
V_{1} U_{3}-U_{1} V_{3}+H_{2} K_{2}-L_{2} \Xi=0, & V_{3} U_{4}-U_{3} V_{4}+H_{2} L_{3}-K_{3} \Xi=0, \\
V_{1} U_{4}-U_{1} V_{4}+H_{2} K_{1}-L_{1} \Xi=0, & V_{4} U_{2}-U_{4} V_{2}+H_{2} L_{2}-K_{2} \Xi=0 .
\end{array}
$$

Consider the invertible linear map

$$
\delta(s)=\left(U(s), V(s), L(s), K(s), H_{2}(s), \Xi(s)\right)
$$

defined by the relations in (5) and (6). Furthermore, let $\mathcal{J}_{0}=\left\{(q, Q) \in \mathbb{R}^{8} \mid \Xi(q, Q)=\right.$ $0,(q, Q) \neq 0\}$. Note that in view of relations (7) and the additional relation $|K|^{2}+$ $|L|^{2}=H_{2}^{2}+\Xi^{2}$, we see that $(q, Q)=(0,0)$ is in one-to-one correspondence with $\left(U, V, L, K, H_{2}, \Xi\right)=(0,0,0,0,0,0)$. Thus, the reduced phase space $\delta \circ \tau_{\Xi}\left(\Xi^{-1}(0)\right)$ consists of more than one symplectic leaf, the origin being one of them.

The space given by Eqs. (7), with $H_{2}=h$ and $\Xi=0$ fixed is $T^{h} S_{h}^{3}$. Furthermore, define

$$
M_{0}=\delta \circ \tau_{\Xi}\left(\mathcal{J}_{0}\right)
$$

that is, $M_{0}$ is the $\Xi$-reduced phase space corresponding to $\Xi=0$ without the origin.

\section{Theorem 1}

(i) $M_{0}$ is in $(U, V)$-space given by the equations $|U|^{2}=|V|^{2}$ and $<U, V>=0,(U, V) \neq$ $(0,0)$.

(ii) $M_{0}$ is diffeomorphic to $T^{1} S_{1}^{3} \times \mathbb{R}_{>0}$.

(iii) $M_{0}$ is diffeomorphic to $T^{+} S^{3}$.

Proof (i) In view of relations (8) and (7), we can express the invariants $K, L$, and $\mathrm{H}_{2}$ in $U, V$ provided $\Xi=0$ and $H_{2} \neq 0$, that is, $(U, V) \neq(0,0)$. It follows that we may represent $M_{0}$ as a subspace of $(U, V)$-space given by the equations $|U|^{2}=|V|^{2}$ and $<U, V>=0,(U, V) \neq(0,0)$.

(ii) Again considering Eq. (7), setting $H_{2}=h>0$, in $\left(U, V, H_{2}\right)$ space we obtain $T^{h} S_{h}^{3} \times \mathbb{R}$ given by $|U|^{2}=|V|^{2}=h^{2}$ and $\left\langle U, V>=0,(U, V) \neq(0,0), h \in \mathbb{R}_{>0}\right.$. By the transformation $(U, V)->\left(U / H_{2}, V / H_{2}\right)$, we obtain $T^{1} S_{1}^{3} \times \mathbb{R}_{>0}$.

(iii) We follow an approach similar to the one in Kummer (1982). Let

$$
\tilde{\delta}(s)=\left(u(s), v(s), L(s), K(s), H_{2}(s), \Xi(s)\right),
$$

with $u=\frac{U}{H_{2}}$, and $v=V$. Then it easily follows that the reduced phase space $M_{0}$ is diffeomorphic to $T^{+} S^{3}$, that is, the reduced phase space $\delta \circ \tau_{\Xi}\left(\mathcal{J}_{0}\right)$ is diffeomorphic to $T^{+} S^{3}$, the tangent bundle to the unit three sphere minus its zero section.

Consequently the reduced phase space $\tau_{\Xi}\left(\Xi^{-1}(0)\right)$ has a stratification into the $H_{2}$ level sets $T^{h} S_{h}^{3}$. 
Let $\{,\}_{U, V}$ denote the Poisson bracket on $(U, V)$-space induced by the standard Poisson bracket on $(q, Q)$-space. It has structure matrix

$$
\left(\begin{array}{cc}
A & 2 H_{2} I_{4} \\
-2 H_{2} I_{4} & A
\end{array}\right) \text { with } A=2\left(\begin{array}{cccc}
0 & -K_{3} & -K_{2} & -K_{1} \\
K_{3} & 0 & -L_{1} & L_{2} \\
K_{2} & L_{1} & 0 & L_{3} \\
K_{1} & -L_{2} & -L_{3} & 0
\end{array}\right),
$$

with $I_{4}$ the $4 \times 4$ identity matrix. If $H_{2} \neq 0$, one can express $K, L, H_{2}$ in terms of $U$ and $V$ using the relations (7) and (8) this gives a bracket on $\mathbb{R}^{8}$ in the coordinates $(U, V)$.

As in Theorem (1), $M_{0}$ is the $\Xi=0$ reduced phase space in $\mathbb{R}^{8}$, with coordinates $(U, V)$, given by the equations $|U|^{2}-|V|^{2}=0$ and $\langle U, V\rangle=0$. Let $\{,\}^{M_{0}}$ denote the constrained bracket, or Dirac bracket Dirac (1950), obtained by constraining the standard Poisson bracket on $(U, V)$-space to $M_{0}$. The following theorem states that the restriction of the standard Poisson bracket on $\mathbb{R}^{8}$ to the image of the orbit map, seen as a manifold in $\mathbb{R}^{8}$, i.e., the constrained bracket, is the same as the bracket induced by the orbit map.

Theorem $2\{,\}_{U, V}=2 H_{2}\{,\}^{M_{0}}$.

Proof Using the formula for the Dirac bracket

$$
\left\{F\left|M_{0}, G\right| M_{0}\right\}^{M_{0}}=\{F, G\}_{U, V}-\Sigma_{i, j=1}^{2}\left\{F, F_{i}\right\}_{U, V} c^{i j}\left\{F_{j}, G\right\}_{U, V},
$$

with $F_{1}(U, V)=|U|^{2}-|V|^{2}, F_{2}(U, V)=<U, V>$ and $c^{i j}$ the inverse of the matrix given by $\left\{F_{i}, F_{j}\right\}_{U, V}$, the result follows by straightforward computation. Note that the $(U, V)$ bracket is induced by the standard bracket when using coordinates $(q, Q)$.

Remark 2 In van der Meer and Cushman (1986), the idea of constrained normalization was introduced in order to normalize perturbed Kepler systems, which were mapped to systems on $T^{+} S^{3}$ by Moser's regularization, by a normalization procedure on the ambient space $\mathbb{R}^{8}$ in a way that had a natural restriction to $T^{+} S^{3}$. Theorem 2 shows that a normalization on the domain of the orbit map, equivariant with respect to the $\Xi$-action, reduces to a constrained normalization on the image of the orbit map in a natural way by the induced bracket.

Remark 3 Note that the map $\delta \circ \tau_{\Xi}$ can also be described in terms of quaternions. If $q$ and $Q$ denote quaternions with the usual base $1, i, j, k$, and with the usual definitions of the quaternion product, quaternion conjugate, norm and inverse, then

$$
\begin{aligned}
& \delta \circ \tau_{\Xi}(q, Q)= \\
& \left(\frac{1}{2}(\bar{q} i q-\bar{Q} i Q), \bar{q} i Q,-\bar{q} Q, \frac{1}{2}(\bar{q} i q+\bar{Q} i Q), \frac{1}{2}|q|^{2}+\frac{1}{2}|Q|^{2}, \frac{1}{2}|q|^{2}-\frac{1}{2}|Q|^{2}\right)= \\
& \left(\left(0, U_{4},-U_{3}, U_{2}\right),\left(-\Xi, V_{4},-V_{3}, V_{2}\right),\left(U_{1}, L_{1}, L_{2}, L_{3}\right),\left(0, K_{1}, K_{2}, K_{3}\right), H_{2}, V_{1}\right)(14)
\end{aligned}
$$

In this, we can recognize the KS1-map $\bar{q} i q$ as defined in Breiter and Langner (2017) as well as the $S O$ (3)-momentum mapping ( $\bar{q} i Q, \frac{1}{2}|q|^{2}+\frac{1}{2}|Q|^{2}, \frac{1}{2}|q|^{2}-\frac{1}{2}|Q|^{2}$ ) (see van der Meer et al. 2016).

\section{Moser's regularization}

In this section, we will reveal the connection between KS and Moser's regularization. We will show that they are connected by a linear mapping on the target space of the orbit map. 
Fig. 2 Diagram involving Moser's map

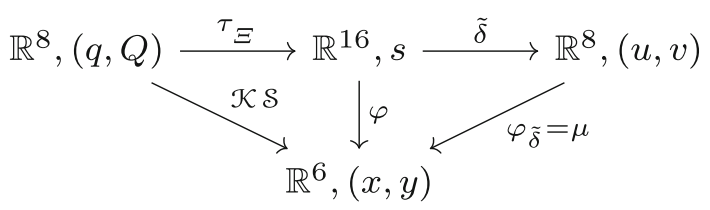

Moser's regularization Moser (1958) connects the Kepler flow to the geodesic flow on $S^{3}$. More precisely, Moser's regularization map maps $T^{+} S^{3}=\left\{(u, v) \in \mathbb{R}^{8}:|u|=1,\langle u, v\rangle=\right.$ $0, v \neq 0\}$ to $\mathbb{R}^{6}$ in such away that the pre-regularized Kepler Hamiltonian $\hat{K}$ maps to the Hamiltonian $|v|$. The orbits of one system are mapped to the orbits of the other system when restricted to the proper energy surface. Note that the system with Hamiltonian $|v|$ still has to be re-scaled in order to obtain the lift of the geodesic flow on $S^{3}$ to $T^{+} S^{3}$ (Cushman and Bates 2015).

In van der Meer (2015), Moser's regularization Moser (1958) was discussed in the framework of reduction following ideas of (Kummer 1982, 1985). In the following, we will give a more extensive treatment showing that there is a direct relation with the KS-regularization, again following the ideas in Kummer (1982). The key is in the diffeomorphism relating the reduced phase space with $T^{+} S^{3}$.

Note that Moser's regularization map maps $T^{+} S^{3}$ to $\mathbb{R}^{6}$, that is, is a coordinate chart for this representation of the $\Xi$-reduced phase space. This chart is constructed by lifting the stereographic projection of $S^{3}$ to the tangent bundle. Also the KS map is a coordinate chart on the reduced phase space. They are related through the map $\tilde{\delta}$ given in (11) as will be shown below.

In the basic set of $\Xi$ invariants given by $\left(U, V, L, K, H_{2}, \Xi\right)$, we have that the KS-chart becomes

$$
\begin{aligned}
2 x_{1} & =U_{2}-K_{3}, & y_{1} & =\frac{V_{2}}{V_{1}+H_{2}}, \\
x_{2} & =U_{3}-K_{2}, & y_{2} & =\frac{V_{3}}{V_{1}+H_{2}}, \\
x_{3} & =U_{4}-K_{1}, & y_{3} & =\frac{V_{4}}{V_{1}+H_{2}} .
\end{aligned}
$$

Theorem 3 The coordinate chart $\varphi_{\tilde{\delta}}$, with $\tilde{\delta}$ given by (11), is Moser's regularization map (see Fig. 2).

Here $\tilde{\delta}$ is essentially Kummer's map as introduced in Kummer (1982).

Proof Recall that Moser's map $\mu: \mathbb{R}^{8} \rightarrow \mathbb{R}^{6} ;(u, v) \rightarrow(x, y)$ is given by

$$
\begin{aligned}
& x_{1}=\left(|v|+v_{1}\right) u_{2}-u_{1} v_{2}, y_{1}=\left(|v|+v_{1}\right)^{-1} v_{2}, \\
& x_{2}=\left(|v|+v_{1}\right) u_{3}-u_{1} v_{3}, y_{2}=\left(|v|+v_{1}\right)^{-1} v_{3}, \\
& x_{3}=\left(|v|+v_{1}\right) u_{4}-u_{1} v_{4}, y_{3}=\left(|v|+v_{1}\right)^{-1} v_{4} .
\end{aligned}
$$

It is based on the inverse stereographic projection of $\mathbb{R}^{3}$ on $S^{3} \subset \mathbb{R}^{4}$. Let $R_{0}=\{(u, v) \in$ $\left.\mathbb{R}^{8}: v_{1}+|v| \neq 0\right\}$. Then $\mu$ is a diffeomorphism of $T^{+} S^{3} \cap R_{0}$ with inverse

$$
\begin{aligned}
& u=\left[|x|\left(1+|y|^{2}\right)\right]^{-1}\left(-2\langle x, y\rangle,\left(1+|y|^{2}\right) x-2\langle x, y\rangle y\right), \\
& v=\left(\frac{1}{2}|x|\left(1-|y|^{2}\right),|x| y\right) .
\end{aligned}
$$


We have, using (8), $\Xi=0$, and $H_{2}=|V|=|v|$

$$
\begin{gathered}
x_{1}=U_{2}-K_{3}=U_{2}-K_{3}+\frac{L_{3} \Xi}{H_{2}}=U_{2}+\frac{V_{1} U_{2}-U_{1} V_{2}}{H_{2}}=\left(|v|+v_{1}\right) u_{2}-u_{1} v_{2}, \\
x_{2}=U_{3}-K_{2}=U_{3}-K_{2}+\frac{L_{2} \Xi}{H_{2}}=U_{3}+\frac{V_{1} U_{3}-U_{1} V_{3}}{H_{2}}=\left(|v|+v_{1}\right) u_{3}-u_{1} v_{3}, \\
x_{3}=U_{4}-K_{1}=U_{4}-K_{1}+\frac{L_{1} \Xi}{H_{2}}=U_{4}+\frac{V_{1} U_{4}-U_{1} V_{4}}{H_{2}}=\left(|v|+v_{1}\right) u_{4}-u_{1} v_{4}, \\
y_{1}=\frac{V_{2}}{V_{1}+H_{2}}=\frac{v_{2}}{v_{1}+|v|}, \\
y_{2}=\frac{V_{3}}{V_{1}+H_{2}}=\frac{v_{3}}{v_{1}+|v|}, \\
y_{3}=\frac{V_{4}}{V_{1}+H_{2}}=\frac{v_{4}}{v_{1}+|v|},
\end{gathered}
$$

which proves the theorem.

The map $(U, V) \rightarrow(u, v)$ given by $u=\frac{U}{H_{2}}, v=V$ considerably messes up the Poisson structure on the image of $\delta \circ \tau_{\Xi}\left(\Xi^{-1}(0)\right)$. However, we have

$$
\left\{U_{i}, H_{2}\right\}=-2 V_{i},\left\{V_{i}, H_{2}\right\}=2 U_{i}, i=1, \ldots, 4,
$$

which does not change when $(U, V)$ is replaced by $(u, v)$. By construction, the $\Xi$-reduced $\mathrm{H}_{2}$ flow leaves $T^{+} S^{3}$ invariant. On $T^{+} S^{3}$ the $\Xi$-reduced $H_{2}$ flow corresponds to the flow of $|v|$ as follows from the second relation in (7). Like in Sect. 3, we have that the flow of $|v|$ in the induced bracket on $T^{+} S^{3}$ is equal to the constrained flow of $|v|$ with respect to the standard Poisson structure on $\mathbb{R}^{8} \supset T^{+} S^{3}$. Thus Moser's map relates the reduced $\mathrm{H}_{2}$ flow to the Kepler flow. The reduced $H_{2}$ flow is on $T^{+} S^{3}$ a re-parametrization of the geodesic flow, while on $S_{H_{2}}^{3} \times S_{H_{2}}^{3}$ it gives a double Hopf-fibration (see van der Meer et al. (2016)).

Remark 4 The $H_{2}$ invariants on the $\Xi$-reduced phase space are generated by the $L_{i}$ and $K_{i}$. It is well known that, by the KS-map, the $L_{i}$ corresponds to the angular momentum vector for the Kepler system and the $K_{i}$ corresponds to the Runge-Lenz or eccentricity vector. In view of the formulas (8) on $(U, V)$-space, we find the $H_{2}$ invariants $M_{i j}=U_{i} V_{j}-U_{j} V_{i}$, $i, j=1,2,3,4, i \neq j$. On $(u, v)$ space, we find the $H_{2}$ invariants $m_{i j}=u_{i} v_{j}-u_{j} v_{i}$, $i, j=1,2,3,4, i \neq j$. Here the $M_{1 j}, m_{1 j}$ corresponds to the eccentricity vector and the $M_{i j}, m_{i j}, i>1$ corresponds to the angular momentum vector, in the chart relating the harmonic oscillator to the Kepler system. For this, we have to restrict to $H_{2}=1$ and $\Xi=0$. Thus, the group of linear symplectic transformations on $(q, Q)$ space leaving $H_{2}$ and $\Xi$ invariant is $S O$ (4). According to Sect. 2, such a map does change the KS-map but leaves the Kepler vector field unchanged. Note that this $S O(4)$ action is not the lift of an $S O(4)$ action on the configuration space. If the action is restricted to the $S O(3)$ action, given by the angular momentum vector the class of KS-maps is obtained that was considered in Breiter and Langner (2017).

\section{Ligon-Schaaf regularization}

The Ligon-Schaaf regularization was introduced in Ligon and Schaaf (1976), based on Ligon (1973), and further studied in (Ligon 2018; Cushman and Duistermaat 1997; Heckman and 
De Laat 2012; Cushman and Bates 2015). Like the Moser regularization, the Ligon-Schaaf regularization is based on the stereographic projection. It maps $\mathbb{R}^{6}$ to $T^{+} S^{3}$, relating the Kepler Hamiltonian for negative energy to the Delaunay Hamiltonian constrained to $T^{+} S^{3}$, which in turn is a re-parametrization of the geodesic flow. The advantage of the Ligon-Schaaf map is that it does not restrict to a single Kepler energy level, but incorporates the Kepler energy in the map. It directly relates the Delaunay Hamiltonian and the Kepler Hamiltonian and avoids time re-scaling. This section is mainly a review based on the above cited literature. However, the factorization of the LS-map is a bit different then the one in Cushman and Bates (2015) because we want to establish the LS-map, or rather the inverse of the LS-map, as a chart for the reduced phase space. That is we have to make sure that everything is expressed in the $\Xi$ invariants used for Moser's map.

The LS-map $\Phi_{L S}$ is given by

$$
\Phi_{L S}(x, y)=(\xi, \eta)=\left(\sin (\zeta) A+\cos (\zeta) B,-\frac{1}{k} \cos (\zeta) A+\frac{1}{k} \sin (\zeta) B\right)
$$

with $-\frac{1}{2} k^{2}$ the Kepler Hamiltonian. Here $A, B$ are the $k$-dependent components of a representation of Moser's map (see Heckman and De Laat 2012) and $T^{+}\left(S^{3}-p\right)$ is $T^{+} S^{3}$ minus the pole used for the stereographic projection in the construction of Moser's map.

Let $P_{-}=\left\{(x, y) \in \mathbb{R}^{6} \mid K(x, y)<0, x \neq 0\right\}$. In Cushman and Duistermaat (1997), we find the following statement which we give here as a proposition.

Proposition 1 The LS-map $\Phi_{L S}$ is completely determined by the following properties:

(i) $\Phi_{L S}$ is an analytic diffeomorphism from $P_{-}$onto $T^{+}\left(S^{3}-p\right)$,

(ii) $\Phi_{L S}$ is a canonical transformation,

(iii) If $\gamma_{K}$ is a solution of the Kepler system in $P_{-}$, then $\Phi \circ \gamma_{K}$ is a solution of the Delaunay vector field in $T^{+} S^{3}$,

(iv) $J=\tilde{J} \circ \Phi$, where $J$ and $\tilde{J}$ are the respective $S O(4)$ momentum mappings on $P_{-}$and $T^{+} S^{3}$.

Consider Moser's map as given by (17). Using $\frac{1}{2}|y|^{2}-\frac{1}{|x|}=-\frac{1}{2}$, we may rewrite this as

$$
\begin{aligned}
& u=\left(-\langle x, y\rangle, \frac{x}{|x|}-\langle x, y\rangle y\right), \\
& v=\left(1-|x||y|^{2},|x| y\right),
\end{aligned}
$$

where $|u|=|v|=1,\left\langle u, v>=0\right.$. Using the scaling $x \rightarrow k^{2} x, y \rightarrow k^{-1} y, K \rightarrow k^{-2} K$, $t \rightarrow k^{3} t, \omega \rightarrow k \omega$ as formulated in Heckman and De Laat (2012) to be able to handle arbitrary negative energy surfaces for the Kepler problem, i.e., introduce the scaling map $\rho$ by $\rho(x, y)=(\tilde{x}, \tilde{y})$, with $\tilde{x}=k^{2} x, \tilde{y}=\frac{1}{k} y$, then the energy surface $K(x, y)=-\frac{1}{2} k^{2}$ is transformed to $K(\tilde{x}, \tilde{y})=-\frac{1}{2}$. The scaled version of Moser's map becomes $\mu^{-1} \circ \rho(x, y)=$ $(\hat{u}, \hat{v})$, with

$$
\begin{aligned}
& \hat{u}=u(\tilde{x}, \tilde{y})=\left(-k\langle x, y\rangle, \frac{x}{|x|}-\langle x, y\rangle y\right)=\left(k u_{1}, u_{2}, u_{3}, u_{4}\right)=\hat{u}(u), \\
& \hat{v}=v(\tilde{x}, \tilde{y})=\left(1-|x||y|^{2}, k|x| y\right)=\left(v_{1}, k v_{2}, k v_{3}, k v_{4}\right)=\hat{v}(v) .
\end{aligned}
$$

Note that, if $K(\tilde{x}, \tilde{y})=-\frac{1}{2}$, we have $|\hat{u}|=|\hat{v}|=1,<\hat{u}, \hat{v}>=0$.

This induces a scaling map

$$
\hat{\rho}: T^{+} S^{3} \rightarrow T_{1} S^{3} \times \mathbb{R}_{>0} ;(u, v) \rightarrow(\hat{u}, \hat{v}, k),
$$


with $(\hat{u}, \hat{v})$ given by (22). We have $\mu^{-1} \circ \rho=\hat{\rho} \circ \mu^{-1}$.

In the LS-map in (20), we now have to choose

$$
\begin{aligned}
& A(x, y)=\hat{u}(x, y)=\left(-k\langle x, y\rangle, \frac{x}{|x|}-\langle x, y\rangle y\right), \\
& B(x, y)=\hat{v}(x, y)=\left(1-|x||y|^{2}, k|x| y\right), \\
& \zeta(x, y)=k<x, y>=-\hat{u}_{1} .
\end{aligned}
$$

Because we are following van der Meer (2015) in our choice of the Moser-map, our formulas differ slightly from the map in Cushman and Duistermaat (1997). However, one can still follow the arguments in Cushman and Duistermaat (1997) to show that this map is actually the LS-map.

We may now unravel the LS-map in a sequence of maps like in Cushman and Bates (2015). First the inverse of Moser's map $\mu^{-1}$ followed by scaling map $\hat{\rho}$. Then the rotation $R_{\hat{u}_{1}}$ over the angle $\zeta=-\hat{u}_{1}$. Combining the last two of these maps gives

$$
R_{\hat{u}_{1}} \circ \hat{\rho}: T^{+} S^{3} \rightarrow T_{1} S^{3} \times \mathbb{R}_{>0} ;(u, v) \rightarrow(\hat{\xi}, \hat{\eta}, k) .
$$

Next consider

$$
\hat{\psi}: T_{1} S^{3} \times \mathbb{R}_{>0} \rightarrow T^{+} S^{3} ;(\hat{\xi}, \hat{\eta}, k) \rightarrow(\xi, \eta) .
$$

As the components of the LS-map are functions of Moser's variables $(u, v)$, we may also introduce

$$
\Psi: T^{+} S^{3} \subset \mathbb{R}^{8} \rightarrow T^{+} S^{3} \subset \mathbb{R}^{8}:(u, v) \rightarrow(\xi, \eta),
$$

given by $\hat{\psi} \circ R_{\hat{u}_{1}} \circ \hat{\rho}$. Then

$$
\Phi_{L S}=\Psi \circ \mu^{-1}=\hat{\psi} \circ R_{\hat{u}_{1}} \circ \hat{\rho} \circ \mu^{-1},
$$

which is illustrated in Fig. 3.

The inverse LS-map $\Phi_{L S}^{-1}$ (see Ligon 2018) now provides a chart for $T^{+} S^{3} \rightarrow \mathbb{R}^{6}$. Following (Cushman and Duistermaat 1997), this mapping maps the Delaunay Hamiltonian to the Kepler problem and is symplectic only if $\zeta(x, y)=k\langle x, y\rangle$. That is the LS-regularization has the advantage that it directly relates not the pre-regularized Kepler Hamiltonian, but the actual Kepler Hamiltonian, to the Delaunay Hamiltonian

$$
D(\xi, \eta)=-\frac{1}{2} \frac{1}{|\eta|^{2}}
$$

on $T^{+} S^{3}$. Note that we actually have to consider the constrained vector field of $D$ on $T^{+} S^{3}$.

Following Cushman and Bates (2015), one obtains that the constrained Delaunay flow on $T^{+} S^{3}$ is a re-parametrized version of the geodesic flow on $T^{+} S^{3}$. Note that this reparametrization can actually be obtained by a symplectic map as the scaling factor is a function of the energy. In this construction, the $\Xi$-reduced vector field corresponding to $D$ using the orbit map $\Psi \circ \tilde{\delta} \circ \tau_{\Xi}\left(\Xi^{-1}(0)\right)$ maps to the Kepler vector filed through the chart given by the inverse LS-map.

For completeness, we will give the inverse LS-map $\Phi_{L S}^{-1}$. We may write $\Phi_{L S}^{-1}$ as a function of $(u, v)$ on the $\Xi$-reduced phase space $\tilde{\delta} \circ \tau_{\Xi}\left(\Xi^{-1}(0)\right)$. One can find the inverse LS-map in Ligon (2018). We have to adjust for our choice of representing Moser's map. The LS-map can be seen as the composition of two maps. The first is the parametrized version of Moser's map given by $\hat{\rho} \circ \mu^{-1}$, the second the rotation in $(u, v)$ space given by $R_{\hat{u}_{1}}$. The inverse 
Fig. 3 Diagram relating Moser's map and the LS-map

$$
\left.\mathbb{R}^{8},(u, v)\right) \stackrel{\Psi}{\longrightarrow} \underset{\left.\mathbb{R}_{L S}\right|^{6},(x, y)}{\mathbb{R}^{8},(\xi, \eta)}
$$

Fig. 4 Commutative diagram involving the KS- and LS-map

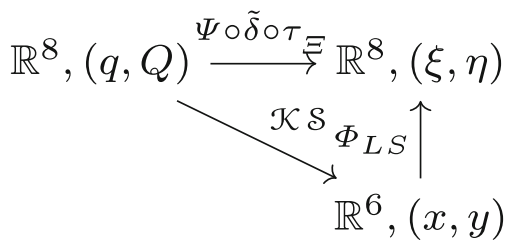

therefore consists of the inverse rotation followed by the inverse of the scaled Moser's map. The inverse of $\hat{\rho} \circ \mu^{-1}$ is given by

$$
x=-\frac{1}{k^{2}}\left(\left(1+v_{1}\right) u-u_{1} v\right), y=\frac{k}{\left(1+v_{1}\right)} v .
$$

Writing $\Phi_{L S}(x, y)=(\xi, \eta)$, the inverse of the rotation $R_{\hat{u}_{1}}$ is given by

$$
u=-\xi \sin (\zeta)+\frac{\eta}{|\eta|} \cos (\zeta), v=\xi \cos (\zeta)+\frac{\eta}{|\eta|} \sin (\zeta)
$$

Substituting (30) in (29) gives

$$
\begin{aligned}
x & =|\eta|^{2}\left(-\xi\left(\frac{\eta_{1}}{|\eta|}+\sin (\zeta)\right)+\frac{\eta}{|\eta|}\left(\xi_{1}+\cos (\zeta)\right)\right) \\
& =|\eta|\left(\xi_{1} \eta-\eta_{1} \xi\right)-|\eta|^{2} \xi \sin (\zeta)+\eta|\eta| \cos (\zeta), \\
y & =\frac{\xi \cos (\zeta)+\frac{\eta}{|\eta|} \sin (\zeta)}{|\eta|\left(1+\xi_{1} \cos (\zeta)+\frac{\eta_{1}}{|\eta|} \sin (\zeta)\right)} .
\end{aligned}
$$

Here we have used the fact that $k=\frac{1}{|\eta|}$. In Ligon (2018), there is an extensive list of relations. Our formulas differ in some signs from the formulas in this paper due to choices made in the stereographic projection. Note that the angle $\zeta$ is now defined implicitly through the relation $\zeta=\xi_{0} \sin (\zeta)-\frac{\eta_{0}}{|\eta|} \cos (\zeta)$ Ligon (2018).

If one chooses $(\xi, \eta)$ as invariants defining the orbit map, then again the $\Xi$-reduced phase space is $T^{+} S^{3}$, and (31) defines a symplectic chart on the reduced phase space. In Fig. 4, a diagram is given showing the relation between the KS-map and the Ligon-Schaaf map. We have a reduction to $T^{+} S^{3}$ using Ligon-Schaaf variables, where the inverse LS-map gives a chart on this representation of $T^{+} S^{3}$.

\section{Reducing the KS-transformed Kepler system}

When one considers the LS-regularization, then the following features make it different. The Kepler Hamiltonian is directly related to the Delaunay Hamiltonian on $T^{+} S^{3}$, no preregularized Kepler Hamiltonian is needed and no time re-scaling. In the Ligon-Schaaf regularization, the Keplerian energy, or actually the Keplerian Hamiltonian, is introduced as a scaling factor for the coordinates. 
In order to understand the role of the Ligon-Schaaf map in the reduction procedure, recall the role of the Keplerian energy in the construction of the pre-regularized Kepler Hamiltonian in Sect. 2 and how it relates to the harmonic oscillator.

By a re-scaling of time, the orbits of the Kepler Hamiltonian for negative energy $-\frac{1}{2} k^{2}$, $k>0$, were obtained as the re-parametrized orbit of the pre-regularized Hamiltonian

$$
\hat{K}(x, y)=\frac{|x|}{k}\left(K(x, y)+\frac{1}{2} k^{2}\right)+\frac{1}{k}=\frac{1}{2 k}|x|\left(|y|^{2}+k^{2}\right)
$$

on $\left(T^{*} \mathbb{R}^{3}, \tilde{\omega}\right)$.

Using the KS-map, we have

$$
|x|=q_{1}^{2}+q_{2}^{2}+q_{3}^{2}+q_{4}^{2},|y|^{2}=\frac{Q_{1}^{2}+Q_{2}^{2}+Q_{3}^{2}+Q_{4}^{2}}{q_{1}^{2}+q_{2}^{2}+q_{3}^{2}+q_{4}^{2}}-\frac{\left(\left(q_{1} Q_{2}-q_{2} Q_{1}\right)+\left(q_{3} Q_{4}-q_{4} Q_{3}\right)\right)^{2}}{\left(q_{1}^{2}+q_{2}^{2}+q_{3}^{2}+q_{4}^{2}\right)^{2}} .
$$

Furthermore,

$$
\Xi(q, Q)=\left(q_{1} Q_{2}-q_{2} Q_{1}\right)+\left(q_{3} Q_{4}-q_{4} Q_{3}\right) .
$$

One finds that the KS-map maps the pre-regularized Kepler Hamiltonian to

$$
\mathcal{K S}^{*} \hat{K}=\frac{1}{2 k}\left(k^{2}\left(q_{1}^{2}+q_{2}^{2}+q_{3}^{2}+q_{4}^{2}\right)+\left(Q_{1}^{2}+Q_{2}^{2}+Q_{3}^{2}+Q_{4}^{2}\right)\right),
$$

when restricted to $\mathcal{J}_{0}$.

Taking $k=1$ the $H_{2}(q, Q)=\frac{1}{2}|q|^{2}+\frac{1}{2}|Q|^{2}=1$ level corresponds to $\hat{K}=1$ and $K=-\frac{1}{2}$.

We will start with considering the relation between the KS-map and the LS-map for $k=1$. Using the KS-map, we may, provided $\Xi=0$, rewrite the Kepler Hamiltonian as

$$
\bar{K}(q, Q)=K \circ \mathcal{K S}(q, Q)=\frac{1}{2} \frac{|Q|^{2}}{|q|^{2}}-\frac{1}{|q|^{2}} .
$$

When we use the orbit map

$$
\delta \circ \tau_{\Xi}: \mathbb{R}^{8} \rightarrow \mathbb{R}^{16}:(q, Q) \rightarrow\left(U, V, K, L, H_{2}, \Xi\right),
$$

the Hamiltonian on the image becomes

$$
\bar{K}(q, Q)=\frac{1}{2} \frac{|Q|^{2}}{|q|^{2}}-\frac{1}{|q|^{2}}=\frac{1}{2} \frac{H_{2}-V_{1}}{H_{2}+V_{1}}-\frac{1}{H_{2}+V_{1}},
$$

where $H_{2}=|V|$. Restricting to $H_{2}=1, \Xi=0$, then we have that

$$
\frac{1}{2} \frac{H_{2}-V_{1}}{H_{2}+V_{1}}-\frac{1}{H_{2}+V_{1}}=-\frac{1}{2} \frac{1}{H_{2}^{2}},
$$

where the functions on the right- and left-hand side are equivalent provided we restrict to $H_{2}=1$ and $|q| \neq 0$. The reduced phase space for the orbit map $\delta \circ \tau_{\Xi}$ is $M_{0}$, as given in (10). $M_{0}$ considered as a manifold in $\mathbb{R}^{8}$, with coordinates $(U, V)$, is diffeomorphic to $T^{+} S^{3}$. There exists a mapping $(U, V) \rightarrow(\xi, \eta)$, where $(\xi, \eta)$ are given by the LS-map with $k=1$, mapping $M_{0}$ to $T^{+} S^{3}$. More precisely, we have $\Psi_{L S}^{-1} \circ \psi \circ \tilde{\delta} \circ \tau_{\Xi}=\mathcal{K} \mathcal{S}$, with $\tilde{\delta}$ given by (11) and $\psi$ given by (27).

Rewriting the Delaunay Hamiltonian

$$
D(\xi, \eta)=-\frac{1}{2} \frac{1}{|\eta|^{2}},
$$


in terms of $(q, Q)$ coordinates, we obtain

$$
-\frac{1}{2} \frac{1}{|\eta(q, Q)|^{2}}=-\frac{1}{2} \frac{1}{H_{2}^{2}(q, Q)}=\bar{K}(q, Q),
$$

that is, the Delaunay Hamiltonian corresponds to the Kepler Hamiltonian, provided we are on the energy levels $\Xi=0, H_{2}=1$, and $K=-\frac{1}{2}$.

Remark 5 To obtain the inverse LS-map for $k=1$, we have to rotate $(U, V)$, using the harmonic oscillator flow, over an angle depending on the position, such that the speed of the Delaunay flow matches the speed of the Kepler flow. According to Cushman and Bates (2015) in the scaling $\frac{\mathrm{d} s}{\mathrm{~d} t}=\frac{k}{|x|}$, the new timescale $s$ is the eccentric anomaly. Furthermore, $\zeta=-\langle x, y\rangle=-\mathrm{e} \sin (s)$, and $s-\mathrm{e} \sin (s)=t-\tau$, where $\tau$ is a time related to the periapse passage.

Thus, the rotation in the LS-map makes the total rotation time of the Delaunay vector field equal to that of the Kepler vector field.

Remark 6 The intertwining of the $S O(4)$ momentum mappings, that plays a role in the LSmap, is immediate. The expressions $U_{i} V_{j}-U_{j} V_{i}, i, j=1, \ldots, 4, i \neq j$, generate the $S O$ (4) momentum map on $(U, V)$ space and correspond to the $(K, L)$ variables, which in van der Meer (2015) were shown to correspond to the $S O(4)$ symmetry of the Kepler problem.

In order to bring the Keplerian energy into the reduction process consider

$$
H_{2, k}(q, Q)=k^{2}\left(q_{1}^{2}+q_{2}^{2}+q_{3}^{2}+q_{4}^{2}\right)+\left(Q_{1}^{2}+Q_{2}^{2}+Q_{3}^{2}+Q_{4}^{2}\right) .
$$

$H_{2, k}=1$ corresponds to $\hat{K}=\frac{1}{k}$ and $K=-\frac{1}{2} k^{2} . H_{2, k}$ can be obtained from $H_{2}$ by the scaling $q \rightarrow k q, Q \rightarrow Q, H_{2} \rightarrow H_{2, k}, \omega \rightarrow k \omega$. More precisely set $\tilde{q}=k q$ and $\tilde{Q}=Q$, then $H_{2, k}(q, Q)=H_{2}(\tilde{q}, \tilde{Q})$. Note that through the $\mathcal{K} \mathcal{S}$-map this gives us the scaling $x \rightarrow k^{2} x$, $y \rightarrow k^{-1} y$, that is, if we set, as before, $\tilde{x}=k^{2} x$, and $\tilde{y}=\frac{1}{k} y$, then we obtain the scaling also used in the Ligon-Schaaf regularization. Set

$$
k=\sqrt{-2 K}=\sqrt{-|y|^{2}+\frac{2}{|x|}}=\sqrt{-\frac{|Q|^{2}}{|q|^{2}}+\frac{2}{|q|^{2}}} .
$$

When this is substituted in $H_{2, k}$, then $H_{2, k}$ becomes identically equal to 1 . Thus, $H_{2}$ drops out of the discussion. In stead of $H_{2}$, we introduce $\bar{K}(q, Q)$ as an invariant and consider the orbit map

$$
\bar{\tau}_{\Xi}: \mathbb{R}^{8} \rightarrow \mathbb{R}^{16} ;(q, Q) \rightarrow(\xi, \eta, \bar{K}, K, L, \Xi),
$$

with $\xi, \eta$ the Ligon-Schaaf variables as in Sect. 5. Then the reduced phase space for $\Xi=0$ is, as before, $T^{+}\left(S^{3}-p\right)$, where the pole of the sphere has to be left out as it is the image of $|q|=0$. The reduced system corresponding to $\bar{K}(q, Q)$ is now the Delaunay system with Hamiltonian $-\frac{1}{2} \frac{1}{|\eta|^{2}}$. As the Ligon-Schaaf map is a symplectic map with standard symplectic forms on $\mathbb{R}^{8}$ and $\mathbb{R}^{6}$, the actual reduced system is the Delaunay system constrained to $T^{+} S^{3}$. If for instance we consider on $\mathbb{R}^{8}$ the system with Hamiltonian $-\frac{1}{2} \frac{1}{\left.|\xi|^{2}|\eta|^{2}-<\xi, \eta\right\rangle^{2}}$, then this system leaves $T^{+} S^{3}$ invariant and the corresponding Hamiltonian flow is on $T^{+} S^{3}$ equivalent to the constrained flow of the Delaunay Hamiltonian. 


\section{Alternative reduction and regularization}

The final chart is symplectic by construction when on $T^{+} S^{3}$ we take the constrained induced bracket. Also all brackets on the reduced phase space can be computed using the Poisson bracket on $(q, Q)$ space where we have the standard symplectic form. Therefore, we may relax the condition that the symplectic form on the ambient space of $T^{+} S^{3}$ is the standard one, which means that also other reductions might be considered. We might for instance perform the reduction on $\mathbb{R}^{8}$ with coordinates $(\tilde{q}, \tilde{Q})$. Because we use $(\tilde{q}, \tilde{Q})$ instead of $(q, Q)$, our scaling is introduced in all mappings. We obtain the invariants $\tilde{s}(s)=s(\tilde{q}, \tilde{Q})$

$$
\tilde{s}=\left(k^{2} s_{1}, k^{2} s_{2}, s_{3}, s_{4}, k s_{5}, k s_{6}, k s_{7}, k s_{8}, k^{2} s_{9}, k^{2} s_{10}, s_{11}, s_{12}, k s_{13}, k s_{14}, k s_{15}, k s_{16}\right) .
$$

The $\tilde{s}$ define again a set of $\Xi$-invariants which we may use to define a $\Xi$-orbit map, which we indicate by $\tilde{\tau}_{\Xi}$. Previously we changed from $s$ invariants to invariants $\left(K(s), L(s), U(s), V(s), H_{2}(s), \Xi(s)\right)$ given by (5) and (6). If we do the same for $\tilde{s}$, we obtain

$$
\begin{gathered}
\tilde{U}(q, Q)=U(\tilde{s}(q, Q))=U(\tilde{q}, \tilde{Q}), \quad \tilde{V}(q, Q)=V(\tilde{s}(q, Q))=V(\tilde{q}, \tilde{Q}), \\
\tilde{K}(q, Q)=K(\tilde{s}(q, Q))=K(\tilde{q}, \tilde{Q}), \quad \tilde{L}(q, Q)=L(\tilde{s}(q, Q))=L(\tilde{q}, \tilde{Q}), \\
\tilde{H}_{2}(q, Q)=H_{2}(\tilde{s}(q, Q))=H_{2}(\tilde{q}, \tilde{Q})=H_{2, k}(q, Q), \quad \tilde{\Xi}(q, Q)=\Xi(\tilde{s}(q, Q))=\Xi(\tilde{q}, \tilde{Q}),
\end{gathered}
$$

where $\tilde{U}_{1}=k U_{1}, \tilde{V}_{2}=k V_{2}, \tilde{V}_{3}=k V_{3}, \tilde{V}_{4}=k V_{4}, \tilde{H}_{2}=H_{2, k}, \tilde{\Xi}=k \Xi$. Furthermore, $k$ is present in $\tilde{U}_{2}, \tilde{U}_{3}, \tilde{U}_{4}$ and $\tilde{V}_{1}$. Thus, $\tilde{U}$ and $\tilde{V}$ are different from the scaled Moser variables. As $H_{2, k}$ becomes identically 1 , we introduce $\bar{K}$ as an invariant. Furthermore, we use $\Xi$ instead of $\tilde{\Xi}$. Consider the orbit map

$$
\tilde{\tau}_{\Xi}: \mathbb{R}^{8} \rightarrow \mathbb{R}^{16} ;(q, Q) \rightarrow(\tilde{U}, \tilde{V}, \tilde{K}, \tilde{L}, \bar{K}, \Xi) .
$$

Relations (7) and (8) still hold for the variables $\left(\tilde{U}, \tilde{V}, \tilde{K}, \tilde{L}, \tilde{H}_{2}, \tilde{\Xi}\right)$. That is, the reduced phase space for $\Xi=0$ is the six dimensional manifold $T^{1} S_{1}^{3} \times \mathbb{R}_{<0}$ in $(\tilde{U}, \tilde{V}, \bar{K})$-space. By introducing

$$
\bar{\rho}: T_{1} S^{3} \times \mathbb{R}_{<0} \rightarrow T^{+} S^{3}:(\tilde{U}, \tilde{V}, \bar{K}) \rightarrow(\bar{U}, \bar{V}),
$$

with $\bar{U}=\tilde{V}$ and $\bar{V}=\frac{1}{k} \tilde{U}$ we obtain the orbit map

$$
\bar{\tau}_{\Xi}: \mathbb{R}^{8} \rightarrow \mathbb{R}^{16} ;(q, Q) \rightarrow(\bar{U}, \bar{V}, \tilde{K}, \tilde{L}, \bar{K}, \Xi) .
$$

The reduced phase space for $\Xi=0$ is the six-dimensional manifold $T^{+}\left(S^{3}-p\right)$ in $(\bar{U}, \bar{V})$ space. In terms of $(q, Q)$ coordinates, we have for the Delaunay Hamiltonian

$$
D(\bar{U}(q, Q), \bar{V}(q, Q))=-\frac{1}{2} \frac{1}{|\bar{V}(q, Q)|^{2}}=\bar{K}(q, Q),
$$

that is, the Delaunay system on $T^{+} S^{3}$ is the reduced KS-transformed Kepler system with Hamiltonian $\bar{K}(q, Q)$.

The chart we have to consider on $T^{+} S^{3}$ is a modification of the chart for the Moser regularization.

$$
\begin{aligned}
& x_{1}=\frac{1}{k}\left(\left(|\bar{U}|+\bar{U}_{1}\right) \bar{V}_{2}-\bar{V}_{1} \bar{U}_{2}\right), y_{1}=k\left(\left(|\bar{U}|+\bar{U}_{1}\right)^{-1} \bar{U}_{2}\right), \\
& x_{2}=\frac{1}{k}\left(\left(|\bar{U}|+\bar{U}_{1}\right) \bar{V}_{3}-\bar{V}_{1} \bar{U}_{3}\right), y_{2}=k\left(\left(|\bar{U}|+\bar{U}_{1}\right)^{-1} \bar{U}_{3}\right),
\end{aligned}
$$




$$
x_{3}=\frac{1}{k}\left(\left(|\bar{U}|+\bar{U}_{1}\right) \bar{V}_{4}-\bar{V}_{1} \bar{U}_{4}\right), y_{3}=k\left(\left(|\bar{U}|+\bar{U}_{1}\right)^{-1} \bar{U}_{4}\right),
$$

where $k=\frac{1}{|\bar{V}|}$.

A similar result can be obtained by simplifying the LS-reduction. Consider the diffeomorphism given by (23)

$$
\hat{\rho}: T^{+} S^{3} \rightarrow T_{1} S^{3} \times \mathbb{R}_{>0} ;(u, v, k) \rightarrow(\hat{u}, \hat{v})
$$

followed by the map $\bar{\rho}$ given in (36). We have the reduction mapping

$$
\bar{\rho} \circ \hat{\rho} \circ \tilde{\delta} \circ \tilde{\tau}_{\Xi}: \mathbb{R}^{8} \rightarrow T^{+} S^{3} \subset \mathbb{R}^{8} ;(q, Q) \rightarrow(\hat{\xi}, \hat{\eta}) .
$$

Consequently on the image of the reduction mapping given by (38), we have the chart given by the inverse of $\bar{\rho} \circ \hat{\rho} \circ \mu^{-1}$.

The Hamiltonian system with Hamiltonian

$$
\bar{K}(q, Q)=K \circ \mathcal{K} \mathcal{S}(q, Q)=\frac{1}{2} \frac{|Q|^{2}}{|q|^{2}}-\frac{1}{|q|^{2}}
$$

reduces to the Delaunay Hamiltonian system on $T^{+} S^{3}$ given by

$$
D(\hat{\xi}, \hat{\eta})=-\frac{1}{2} \frac{1}{|\bar{V}|^{2}},
$$

where now $(\bar{U}, \bar{V})=\bar{\rho} \circ \hat{\rho}(u, v)$. The Delaunay system in the above chart maps to the Kepler system.

We replaced the factor $\hat{\psi} \circ R_{\hat{u}_{1}} \circ \hat{\rho}$ of the LS-map by $\bar{\rho} \circ \hat{\rho}$ obtaining yet another reduction map and another representation of $T^{+} S^{3}$. The map $\mathbb{R}^{8},(\bar{U}, \bar{V}) \rightarrow \mathbb{R}^{6},(x, y)$ is again the chart on $T^{+} S^{3}$ in which we obtain the Kepler system. Like for the LS-map in Sect. 6 in this case the reduction to $(\bar{U}, \bar{V})$ variables gives a regularization for all negative energy levels of the Kepler system.

Furthermore, if we choose $\bar{U}=\tilde{V}$, and $\bar{V}=k \tilde{U}$ the reduced Hamiltonian will become $-\frac{1}{2}|\bar{V}|^{2}$. Note that in this case we may replace the reduced Hamiltonian $-\frac{1}{2}|\bar{V}|^{2}$ on $T^{+} S^{3}$ by $-\frac{1}{2}\left(|\bar{V}|^{2}|\bar{U}|^{2}-<\bar{V}, \bar{U}>^{2}\right)$. As a consequence, the normalization and analysis of perturbed Keplerian systems as in van der Meer and Cushman (1986); van der Meer (1988); Cushman (1992) seems to apply for arbitrary negative Keplerian energy. This will be subject of further research.

To obtain results for positive Keplerian energy, we may adapt our invariants in a way similar to Kummer (1982); Ligon (2018). Again choose invariants $\tilde{s}$ but take

$$
k=\sqrt{2 K}
$$

and replace $k^{2}$ by $-k^{2}$. Then our relations for $\tilde{U}$, and $\tilde{V}$ become

$$
\begin{aligned}
<\tilde{U}, \tilde{V}> & =0, \\
-\tilde{U}_{1}^{2}+\tilde{U}_{2}^{2}+\tilde{U}_{3}^{2}+\tilde{U}_{4}^{2} & =1, \\
\tilde{V}_{1}^{2}-\tilde{V}_{2}^{2}-\tilde{V}_{3}^{2}-\tilde{V}_{4}^{2} & =1 .
\end{aligned}
$$

With the additional map $\bar{U}=\tilde{V}$, and $\bar{V}=k \tilde{U}$ one gets the reduced system with Hamiltonian $\frac{1}{2}\left(-\bar{V}_{1}^{2}+\bar{V}_{2}^{2}+\bar{V}_{3}^{2}+\bar{V}_{4}^{2}\right)$ on the reduced phase space $T^{+} H^{3}$ given by (39).

Acknowledgements Parts of the results in this paper were presented by the author during the II Workshop on Hamiltonian Dynamical Systems and Celestial Mechanics, Jan.13-17, 2020, Universidad del Bío-Bío, Chile. 
Open Access This article is licensed under a Creative Commons Attribution 4.0 International License, which permits use, sharing, adaptation, distribution and reproduction in any medium or format, as long as you give appropriate credit to the original author(s) and the source, provide a link to the Creative Commons licence, and indicate if changes were made. The images or other third party material in this article are included in the article's Creative Commons licence, unless indicated otherwise in a credit line to the material. If material is not included in the article's Creative Commons licence and your intended use is not permitted by statutory regulation or exceeds the permitted use, you will need to obtain permission directly from the copyright holder. To view a copy of this licence, visit http://creativecommons.org/licenses/by/4.0/.

\section{References}

Breiter, S., Langner, K.: Kustaanheimo-Stiefel transformation with an arbitrary defining vector. Celest. Mech. Dyn. Astron. 128, 323-342 (2017)

Cordani, B.: The Kepler Problem. Springer, Basel (2003)

Cushman, R.H., Duistermaat, J.J.: A Characterization of the Ligon-Schaaf Regularization Map, Communications on Pure and Applied Mathematics, Vol. L, 773-787 (1997)

Cushman, R.H.: A survey of normalization techniques applied to perturbed Keplerian systems. In: Jones, C.K.R.T., Kirchgraber, U., Walther, H.O. (eds.) Dynamics Reported, vol. 1, pp. 54-112. Springer, Berlin (1992)

Cushman, R.H., Bates, L.M.: Global aspects of classical integrable systems, 2nd edn. Springer Basal, Berlin (2015)

Deprit, A., Rom, A.: The main problem of artificial satellite theory for small and moderate eccentricities. Celest. Mech. 2, 166-206 (1970)

Deprit, A., Elipe, A., Ferrer, S.: Linearization: Laplace vs Stiefel. Celest. Mech. Dyn. Astron. 58, 151-201 (1994)

Dirac, P.: Generalized hamiltonian dynamics. Canadian J. Math. 2, 129-148 (1950)

Efstathou, K., Sadovskii, D.A.: Normalization and global analysis of the hydrogen atom. Rev. Mod. Phys. 82, 2099-2154 (2010)

Egea, J.: Sistemas Hamiltonianos en resonancia 1:1:1:1. Tesis Doctoral, Universidad de Murcia, Reducciones toroidales y bifurcaciones de Hopf (2007)

Ferrer, S., Crespo, F.: Alternative angle-based approach to the KS-Map. An interpretation through symmetry and reduction. J. Geometric Mech. 10, 359-372 (2018)

Heckman, G., De Laat, T.: On the regularization of the Kepler problem. J. Symplectic Geom. 10, 463-473 (2012)

Kummer, M.: On the regularization of the Kepler problem. Commun. Math. Phys. 84, 133-152 (1982)

Kummer, M.: A Group Theoretical Approach to a Certain Class of Perturbations of the Kepler Problem. Arch. Ration. Mech. Anal. 91, 55-82 (1985)

Kustaanheimo, P.: Spinor regularization of the Kepler motion Ann. Univ. Turku. Ser. A I, 73, 1-7 (1964) Also: Publications of the Astronomical Observatory Helsinki, 102 (1964)

Kustaanheimo, P., Stiefel, E.: Perturbation theory of Kepler motion based on spinor regularization. J. für die reine und angewandte Mathematik 218, 204-219 (1965)

Levi-Civita, T.: Sur la résolution qualitative du problème restreint des trois corps. Acta Math. 30, 305-327 (1906)

Ligon, T.S.: Das Keplerproblem differentialgeometrisch gesehen. Ludwig-Maximilians-Universität, München, Studienabschlussarbeit (1973)

Ligon, T.S.: The symmetry of the Kepler problem, the inverse Ligon-Schaaf mapping and the Birkhoff conjecture. PlosOne 13, e0203821 (2018). https://doi.org/10.1371/journal.pone.0203821

Ligon, T.S., Schaaf, M.: On the global symmetry of the classical Kepler problem. Rep. Math. Phys. 9, 281-300 (1976)

Moser, J.: Regularization op Keplers problem and the averaging method on a manifold. Comm. Pure Appl. Math. 23, 609-636 (1958)

Saha, P.: Interpreting the Kustaanheimo-Stiefel transform in gravitational dynamics. Mon. Not. R. Astron. Soc. 400, 228-231 (2009)

Stiefel, E.L., Scheifele, G.: Linear and regular celestial mechanics: perturbed two-body motion, numerical methods, canonical theory, Grundlehren der mathematischen Wissenschaften, 174. Springer-Verlag, Berlin Heidelberg (1971)

van der Meer, J.C. : Integrability and reduction of perturbed Keplerian systems, RANA report 88-15, Eindhoven University of technology (1988) 
van der Meer, J.C.: The Hamiltonian Hopf bifurcation, LNM 1160. Springer Verlag, Berlin (1985)

van der Meer, J.C.: The Kepler system as a reduced 4D harmonic oscillator. J. Geom. Phys. 92, 181-193 (2015)

van der Meer, J.C., Cushman, R.H.: Constrained normalization of Hamiltonian systems and perturbed Keplerian motion. J. Appl. Math. Phys. 37, 402-424 (1986)

van der Meer, J.C., Crespo, F., Ferrer, S.: Generalized Hopf fibration and geometric SO(3) reduction of the 4DOF harminic oscillator. Rep. Math. Phys. 77, 239-249 (2016)

Vivarelli, M.D.: The KS-transformation in hypercomplex form. Celest. Mech. 29, 45-50 (1983)

Waldvogel, J.: Quaternions and the perturbed Kepler problem. Celest. Mech. Dyn. Astron. 95, 201-212 (2006)

Publisher's Note Springer Nature remains neutral with regard to jurisdictional claims in published maps and institutional affiliations. 